\title{
Individual Differences and Expectations of Automated Vehicles
}

Qiaoning Zhang

School of Information, University of Michigan, Ann Arbor, USA

qiaoning@umich.edu

X. Jessie Yang

Department of Industrial \& Operations Engineering, University of Michigan, Ann Arbor, USA

Lionel P. Robert Jr.

School of Information, University of Michigan, Ann Arbor, USA

Zhang, Q., Yang, X. J. and Robert, L. P. (2021). Individual Differences and Expectations of Automated Vehicles, International Journal of Human-Computer Interaction, Accepted. 


\section{Individual Differences and Expectations of Automated Vehicles}

Despite the benefits of automated vehicles (AVs), there are still barriers to their widespread adoption. Expectations about AVs have been identified as one of the most important factors in understanding AV adoption. Therefore, by understanding the public's expectations of AVs, we can better understand whether or when AVs are likely to be adopted on a wide scale. Individual differences, including demographics and personality, have been identified as factors that impact technology expectations and adoption. However, it is not clear whether and how individual differences can influence expectations of AVs. To examine this, we conducted an online survey with 443 U.S. drivers who were recruited and divided into subpopulations by age, gender, ethnicity, census region, educational level, marital status, income, driving frequency, driving experience, and personality traits. Results revealed that drivers' expectations of AVs differ significantly by age, gender, ethnicity, education levels, marital status, drive frequency, drive experience, and personality. More specifically, higher expectations are more often generated by drivers who are younger, men, White non-Hispanic, more highly educated, never married, with a higher frequency of driving, with less driving experience, and who are high in extraversion, agreeableness, conscientiousness, and emotional stability. The results of this study provide a foundation for future research related to expectations and have important implications on future design and development of AVs.

Keywords: Automated Vehicles, Expectation, Individual Difference, Demographics, Personality, U.S. Drivers, Human-Computer Interaction, Human Robot-Interaction

\section{Introduction}

Automated vehicles (AVs) can benefit society by reducing automobile crashes and saving lives as well as reducing fuel consumption and environmental pollution (Biondi, Alvarez, \& Jeong, 2019; Du et al., 2019; Q. Zhang, Yang, \& Robert, 2021). Despite the potential benefits of AVs, the public is still reluctant to adopt them (Azevedo-Sa et al., 2021; Jayaraman et al., 2019; Nordhoff, van Arem, \& Happee, 2016; Petersen, Robert, Yang, \& Tilbury, 2019; Tan et al., 2021). There are even serious doubts about whether the public will ever adopt AVs on a wide scale. A nationwide survey of 2,167 Americans indicated that $58.4 \%$ were afraid to ride in an AV and only $19.5 \%$ would be 
comfortable riding in an AV (Bansal \& Kockelman, 2017). Because public opinion ultimately plays a crucial role in the adoption of AVs, understanding the factors impacting people's opinions is extremely important (Azevedo-Sa et al., 2020; Detjen et al., 2021; Du et al., 2020; Favarò et al., 2019; Ricci, n.d.).

Expectations are vital to understanding whether users choose to adopt a technology (Bhattacherjee, 2001; Choi \& Ji, 2015; Mamun, Senn, Peak, Prybutok, \& Torres, 2020; Thong, Hong, \& Tam, 2006). Expectations are beliefs about the future performance of a given technology (Borup, Brown, Konrad, \& Van Lente, 2006). Expectation-Confirmation Theory (ECT) posits that whether a product or service failed to meet, met, or exceeded a customer's initial expectations helps to determine their purchase satisfaction and ultimately future purchase intentions (Hossain \& Quaddus, 2012; Michalco, Simonsen, \& Hornbæk, 2015; Oliver, 1980). If expectations are too high, people set themselves up for disappointment after using the technology, which can ultimately lead them to distrust and reject the technology (Lankton, McKnight, \& Thatcher, 2014). When expectations are too low, it can discourage people from ever using the technology at all (Laumer \& Eckhardt, 2012). Thus, an appropriate expectation is crucial to encourage people to drive with an $\mathrm{AV}$ and decrease disappointment by matching/exceeding expectations with actual experience. Therefore, by understanding when AV expectations differ for specific individuals we might be able to set appropriate AV expectations for specific individuals or design AVs to meet or exceed expectations for specific individuals.

Expectations regarding some technologies have been shown to differ significantly among individuals, yet we know very little about whether or how such differences manifest themselves relative to AVs. The term "individual differences" includes traits such as demographics and personality (Harrison \& Rainer, Jr., 1992; Robert, 2018; Robert et al., 2020). Prior research has examined the impact of individual differences on technology adoptions (Esterwood et al., 2021a; Ma et al., 2021). Using the technology acceptance model, Agarwal and Prasad (1999) found that individual differences influence technology acceptance via their effects on individual beliefs about technology. Likewise, research on AVs has shown that individual differences impact AV adoption. For example, several studies have found that women have more concerns about AVs and are less likely to adopt them than men (Schoettle \& Sivak, 2014). Age has also been identified as an important individual difference in the adoption of AVs. For instance, younger drivers have been more likely to adopt AVs than older drivers (Meyer, Becker, Bösch, \& Axhausen, 2017). However, 
the question of whether individual differences influence $\mathrm{AV}$ expectations has received little, if any, attention. This is surprising when we consider the importance of expectations on the adoption of technology in general and the adoption of AVs specifically.

Given the gap and importance of this topic, we conducted an online survey to investigate whether individual differences are related to AV expectations. This national survey consisted of a representative sample of 443 U.S. drivers. The survey collected data on each participant's age, gender, race and ethnicity, education, income, marital status, geographic region, driving frequency, and driving experience. The study examined individual differences in AV expectations based on these key characteristics. Our results have important implications for both research on the adoption of AVs and the design of AVs.

The rest of this paper is organized as follows. Section 2 presents the background for the work. Section 3 describes the details of the study, including the data collection method and sample information. Section 4 presents the survey results. Section 5 discusses the findings in relation to the existing literature. In Section 6, we present the limitations and potential research directions. Finally, the conclusion is presented in Section 7.

\section{Background}

In this section, we summarize the existing literature that informed and motivated our research. First, we present the literature that focused on the role that expectations play on technology adoption by emphasizing its impact on AVs. In doing this, we define AVs as automated driving technologies that encompass SAE level 5 autonomy, which can do all the driving in all circumstances with no human intervention. We then present prior work on individual differences, including personality and demographics, as they relate to AVs. Specifically, we discuss prior research investigating the effects of individual differences on public concerns about adopting AVs.

\subsection{Expectations and Technology Adoption}

The importance of expectations has been greatly emphasized concerning consumer satisfaction and adoption of technology. In this paper, expectations are defined as beliefs about the future performance of a given technology (Borup et al., 2006). The Expectation-Confirmation Theory (ECT), also known as Expectation-Disconfirmation Theory (EDT), provides an explanation on the impacts of expectations on technology adoption. The ECT was initially used to understand 
consumer satisfaction with a given product (Hossain \& Quaddus, 2012; Thong et al., 2006). Generally, customers were more satisfied with a product when it met or exceeded their expectations and were less satisfied with the product when it failed to meet their expectations. One implication of this finding is that setting initial expectations is vital to promoting consumer satisfaction (Michalco et al., 2015; Oliver, 1980; Thong et al., 2006). Research on technology adoption has found similar results: when technology performance exceeds expectations, users are much more likely to adopt that technology (Bhattacherjee, 2001; Brown, Venkatesh, \& Goyal, 2012; Maruping, Bala, Venkatesh, \& Brown, 2017; Sarkar \& Khare, 2019).

Researchers have also examined the impacts of expectations on the intention to adopt AVs. For example, Tussyadiah, Zach, and Wang (2017) conducted a survey study with 325 U.S. residents and found that the likelihood of using AVs (e.g., self-driving taxis) was positively associated with their expectations of the AVs' reliability, functionality, and helpfulness. Similarly, Ro and Ha (2019) examined 1,506 survey responses from South Korea to identify relationships among expectations, attitudes, and behavioral intentions. Ro and Ha found that expectations are positively associated with attitude toward using an $\mathrm{AV}$, which in turn is positively associated with intention to use. Körber, Baseler, and Bengler (2018) examined and found that AV expectations are associated with trust in an automated driving system; they also found that trust in an automated driving system is positively correlated with reliance on the automated driving system.

Expectations have also been employed in broader technology adoption theories including the technology adoption model and the unified theory of acceptance and use of technology (UTAUT). For example, Kaur and Rampersad (2018) surveyed 101 responses to examine the effects of key factors (e.g., performance expectancy, reliability, security, and privacy) and found that expectations positively influence the adoption of AVs. Similar to Kaur and Rampersad, researchers Madigan, Louw, Wilbrink, Schieben, and Merat (2017) surveyed 315 respondents from the city of Trikala, Greece, and applied UTAUT to investigate the factors that influence users' acceptance of AVs (i.e., automated road transport systems). Results provided evidence that expectations regarding performance have a significant impact on intention to use AVs. The results also indicated that effort expectations was not a pivotal factor to impact the intention to use, suggesting that $\mathrm{AV}$ adoption is unlikely to be influenced by the effort required to operate AVs. In all, the existing literature has consistently found a strong link between AV expectations and attitudes toward and the adoption of AVs. 


\subsection{Individual Differences and Automated Vehicles}

Individual differences are the enduring psychological characteristics that distinguish one person from another and help define a person's individuality (Cooper, 2002). Individual differences have been linked to the adoption of various technologies across many settings (Clark et al., 2016; Harrison \& Rainer, Jr., 1992; Im, Bayus, \& Mason, 2003; H.-J. Lee, Jeong Cho, Xu, \& Fairhurst, 2010; Meuter, Bitner, Ostrom, \& Brown, 2005). Demographics and personality traits have been used to represent individual differences (H.-J. Lee et al., 2010; Robert et al., 2020). Various studies have assessed the impact of individual differences on AV acceptance. Next, we present and discuss the literature on individual differences and AV adoption.

Age is among the most important individual differences in predicting AV acceptance. The AV literature found that older drivers generate more negative attitudes and reluctance to adopt AVs. For example, Schoettle and Sivak (2014) conducted a survey with 1,533 respondents from the U.S., the U.K., and Australia to understand their opinions and concerns with accepting AVs. The results indicated that younger drivers are less concerned about AVs, more interested in having AV technology on their vehicle, and more likely to ride in AVs than older drivers. Older drivers also tend to distrust AVs, while younger drivers have shown higher trust in AVs and higher intention to use AVs (Esterwood et al., 2021; Frison et al., 2018; Koul et al., 2018). Driver's age has an important role in understanding the impact of AV explanation on AV trust. Zhang et al. (2021) found that older drivers had higher trust in an AV when it asked for permission to take action, while for younger and middle-aged drivers this actually lowered trust and increased their anxiety. They also found that younger drivers have the lowest anxiety when the AV provided explanations after it took action. On the contrary, this condition produced the highest level of anxiety for middle-aged and older drivers (Zhang et al., 2021).

Gender is a prominent factor in predicting whether someone accepts AV. For instance, Nordhoff, De Winter, Kyriakidis, Van Arem, and Happee (2018) obtained 7,755 survey responses from 116 countries to investigate the determinants of acceptance of driverless shuttles. Their results revealed that men are much more willing to accept driverless shuttles than women. Esterwood et al. (2021) conducted an online survey with 428 participants to understand the impact demographic differences on the acceptance of autonomous buses. The results showed that males expressed a higher intention to ride an autonomous bus than females. They also found that females 
were more concerned about self-driving vehicles and are less likely to believe in their potential benefits than men (Esterwood et al., 2021).

Race and ethnicity have also been investigated as a determinant of AV acceptance. Prior research has been conducted to examine the relationship between race/ethnicity and AV-related attitudes and adoption. For example, Howard and Dai (2014) found that individuals who selfidentified as Hispanic and Asian tend to value AVs' potential to improve mobility for people with driving impairments. Asian Americans had a significantly higher positive attitude towards autonomous buses than White Americans. Another study found that Asian Americans also had a significantly higher positive attitude toward and expressed greater intention to ride autonomous buses than those identifying as White Americans (Esterwood et al., 2021).

Education level is closely associated with AV acceptance. For instance, Schoettle and Sivak (2014) found that higher education levels are associated with the intention to adopt AV technology. Individuals with higher education levels were found to be more likely to have advanced driving technology on their vehicle, more likely to say they would read or work while using AVs, and less likely to say that they would not ride in an AV (Schoettle \& Sivak, 2014).

Prior research also explored the role of income in influencing AV acceptance. Howard and Dai's (2014) survey study found that people with lower income are more concerned with safety issues and giving up control while higher-income drivers pay more attention to liability. Marital status also has an impact on attitudes toward AVs. Results of Howard and Dai's (2014) study suggested that married people are less concerned with cost and amenities (e.g., ability to text message or multitask while driving) but place high importance on safety.

Driving experience and frequency are two individual differences that play roles in understanding the acceptance of advanced driving-related technology like AVs. Koul and Eydgahi (2018) found a negative relationship between driving experience and AV adoption. In Koul and Eydgahi, drivers' intention to use an AV decreased slightly as their years of driving experience increased. Driving frequency has also been found to be negatively associated with AV acceptance. Rödel, Stadler, Meschtscherjakov, and Tscheligi (2014) conducted a survey and found that frequent drivers prefer unassisted driving to technology-assisted driving. Frequent drivers described using the assisted vehicle technology as more challenging and less controllable than people who drove less often. Frequent bus riders had a higher positive attitude and intention to ride autonomous buses than in-frequent riders (Esterwood et al., 2021). 
Geographic region, which has often been used as a proxy for differences in prevailing values and belief systems, has also been identified as a factor that impacts the acceptance of driving technology. Carley, Krause, Lane, and Graham (2013) conducted a survey study in large U.S. cities to examine the intention to purchase plug-in electric vehicles. The results noted a significant difference in electric vehicle adoption across major cities in the United States. Also, the purchase intention of alternative-fuel vehicle technologies differed by geographic regions due again to potential differences in culture, values and beliefs (Pettifor, Wilson, Axsen, Abrahamse, \& Anable, 2017; Sovacool, Kester, Noel, \& de Rubens, 2019). However, geographic region's impact on attitudes toward and adoption of AVs still needs further investigation.

Another class of individual differences - personality — is also associated with AV adoption. Personality is defined as "generalized and personalized determining tendencies-consistent and stable modes of an individual's adjustment to his environment," which can be used as a label to describe traits that represent an individual's predisposition toward behavior or objects (Allport \& Odbert, 1936). The Big Five is the most popular set of personality traits used across many domains, providing a comprehensive taxonomy of individual differences. The Big Five personality model includes openness to experience, conscientiousness, extraversion, agreeableness, and emotional stability (Digman, 1997). Openness to experience represents the flexibility of thought and tolerance of new ideas (McCrae \& Costa, Jr., 1997). Conscientiousness reflects self-control and a need for achievement and order (O'Neill \& Allen, 2011). Extraversion is the extent to which an individual is assertive, outgoing, talkative, and sociable (Digman, 1997). Agreeableness represents the extent to which someone is kind, considerate, likable, and cooperative (Peeters, Van Tuijl, Rutte, \& Reymen, 2006). Emotional stability is the degree to which someone is well-adjusted, emotionally stable, and secure (Peeters et al., 2006).

Kyriakidis, Happee, and de Winter (2015) investigated the relationship between personality traits and concerns over fully AVs. They found that respondents who scored high on emotional stability or lower on agreeableness were more likely to believe that automation was less silly and were also less comfortable with AV data transmission. In addition, T. Zhang et al. (2020) investigated the role of social and personal factors in AV acceptance using a questionnaire survey with 647 drivers in China. Results indicated that drivers with an openness to new experience are more likely to trust and accept AVs. On the contrary, drivers low in emotional stability tended to have distrustful attitudes and lower intentions to use AVs (T. Zhang et al., 2020). 
To summarize, individual differences (i.e., demographics and personality) have significant impacts on attitudes toward and adoption of AVs. However, the literature offers little insight into the role of individual differences associated with $\mathrm{AV}$ expectations and AV adoption although a strong link between the two has been found (Tussyadiah, Zach, \& Wang, 2017; Körber, Baseler, \& Bengler, 2018; Ro \& Ha, 2019).

\section{Method}

To examine whether individual differences impact AV expectations, we conducted a nationwide representative survey with 443 participants using an online survey platform. This research complied with the American Psychological Association code of ethics and was approved by the university's institutional review board. All participants provided informed consent.

\subsection{Survey Instrument and Respondents}

We conducted a survey using an online survey platform in the period of July-September 2018. We developed and distributed a questionnaire for the target study population on www.qualtrics.com. Each respondent's key demographics and individual differences including age, gender, race and ethnicity, education, income, marital status, census region, frequency of driving, driving experience, and personality, along with his/her expectations of AVs, were collected for further analysis. All recorded information was anonymous.

The study population targeted U.S. drivers because the United States is one of the countries expected to be an early adopter of AVs in hopes of achieving greater safety and environmental benefits (Du et al., 2019; Robert, 2019). To select a representative sample of U.S. drivers, we used the Qualtrics Online Sample tool to recruit participants (Boas, Christenson, \& Glick, 2018). There were four steps to obtain a research sample that could represent the characteristics of U.S. drivers. The first step, according to the 2014-2015 statistics of the U.S. Department of Transportation and the AAA Foundation (Triplett, Santos, Rosenbloom, \& Tefft, 2016), was to collect the percentages of subpopulations based on demographics involving age (18+), gender, region, and ethnicity. For the next step, we calculated the study sample size based on a $95 \%$ confidence level, $5 \%$ confidence interval, $50 \%$ population proportion, and total number of U.S. drivers (225 million), which is driven by the population's size reported by the U.S. Census Bureau. The third step was to calculate the numbers of qualified U.S. drivers based on each studied demographic percentage and sample 
size and to provide it to the Qualtrics sample service. Finally, the Qualtrics online sample service selected and included participants within the bounds of the criteria by the embedded screener and collected data from a random and representative sample.

A total of 443 respondents filled out the survey completely. The Qualtrics Online Sample service filtered out partial responses and participants who declined to consent to the study process. Each qualified respondent was paid $\$ 3$ for their participation and responses. A demographics breakdown for the respondents is presented in Table 1.

\subsection{Dependent Variable}

To measure people's expectations of AVs, we used a questionnaire developed by Van Ryzin (2004) with a 7-point Likert rating scale ranging from 1 (low) to 7 (high) (Häuslschmid, von Buelow, Pfleging, \& Butz, 2017). There were three items in the questionnaire: (1) How would you rate your overall expectations regarding the driving of a self-driving car?; How would you rate your expectations regarding the effectiveness of a self-driving car?; and (3) How would you rate your expectations regarding the safety of a self-driving car?

\section{Results}

Our overall objective was to use AV expectations to help identify potential barriers to the adoption of AVs by specific subgroup populations. Therefore, our analysis was designed to detect subgroup population differences based on demographics across our representative sample. Contrary, we did not attempt to build a general predictive model which might fail to detect subgroup differences in smaller subgroups. Nonetheless, our results may help others explore and build valid predictive models.

To accomplish our overall objective, a statistical analysis was performed using IBM SPSS Statistics software. One-way analysis of variance (ANOVA) was used to examine the potential differences in expectation of AVs based on individual differences. The alpha level was set at 0.05 for all statistical tests. All post hoc comparisons utilized a Bonferroni alpha correction. The construct reliability of initial expectation, 0.94 , was above the acceptable threshold of 0.70 . Also, we applied convergent validity through exploratory factor analysis to determine whether this 
measurement construct was valid. All three items loaded above the 0.7 threshold. The summary of responses is shown in Table 2.

\subsection{Age and expectations of $A V s$}

Respondents were divided into two age groups: younger (18-54 years) and older drivers (55 years and older) based on previous age categorizations (C. Lee \& Coughlin, 2015; Waller, 1991). ANOVA revealed a statistically significant effect of people's age on their expectations of AVs (F $\left.=208.856, p<.001, \eta^{2}=.106\right)$. Compared to older drivers (mean $=3.04$, standard deviation [SD] $=1.86)$, younger drivers $($ mean $=4.40, \mathrm{SD}=1.93)$ tended to have higher expectations of AVs.

\subsection{Gender and expectations of $A V S$}

Gender was significant $\left(F=69.818, p<.001, \quad \eta^{2}=.038\right)$. Male drivers $($ mean $=4.31, \mathrm{SD}=1.98)$ had higher expectations of AVs than female drivers (mean $=3.52, \mathrm{SD}=1.96)$.

\subsection{Geographic region and expectations of $A V S$}

Expectations were not significantly different among drivers of different regions (e.g., Northeast, West, South, Midwest $)\left(\mathrm{F}=.657, p=.578, \eta^{2}=.001\right)$. Drivers who lived in the West had the lowest mean $($ mean $=3.79, \mathrm{SD}=2.03)$ compared to drivers in the other three regions.

\subsection{Ethnicity and expectations of $A V s$}

Ethnicity was significant $\left(\mathrm{F}=14.944, p<.001, \eta^{2}=.025\right)$. As illustrated in Figure 1 , post hoc analysis indicated that White non-Hispanic participants tended to have lower expectations than people in the other three ethnic groups: White non-Hispanic (mean $=3.68, \mathrm{SD}=1.99$ ) vs. Black/African American non-Hispanic (mean = 4.40, $\mathrm{SD}=1.94, p<.001)$; White non-Hispanic vs. Hispanic $($ mean $=4.32, \mathrm{SD}=2.07, p<.001)$; White non-Hispanic vs. Other (mean $=4.21$, SD $=1.83, p=.047)$. There was no significant difference among the other three groups.

\subsection{Education and expectations of $A V s$}

Educational level was also significant $\left(\mathrm{F}=5.192, p=.001, \eta^{2}=.009\right)$. As illustrated in Figure 2, post hoc analysis revealed that college-educated drivers had higher expectations of AVs (mean $=$ 
$3.98, \mathrm{SD}=1.95)$ than drivers with some college education ( $\operatorname{mean}=3.58, \mathrm{SD}=2.09, p=.002)$ and those with high school/GED or less education (mean $=3.66, \mathrm{SD}=2.00, p=.022$ ). However, there was no difference between high school graduates and those with some college.

\subsection{Marital status and expectations of AVs}

As shown in Figure 3, there was a significant effect of drivers' marital status on their expectations of AVs $\left(\mathrm{F}=20.319, p<.001, \eta^{2}=.044\right)$. Post hoc comparisons revealed that drivers who had not married had higher AV expectations (mean $=4.47, \mathrm{SD}=1.84$ ) than married drivers (mean $=3.74$, $\mathrm{SD}=2.06, p<.001)$, widowed drivers (mean $=3.25, \mathrm{SD}=1.80, p<.001)$, and divorced $/$ separated drivers (mean $=3.37, \mathrm{SD}=1.94, p<.001)$. There was no difference between never-married drivers and drivers who lived with a partner.

\subsection{Income and expectations of $A V S$}

Participants were divided into three groups by income (i.e., low: $\$ 0-\$ 34,999$; medium: $\$ 35,000$ $\$ 99,999$; high: $\$ 100,000$ or more). There were no significant differences based on income ( $\mathrm{F}=$ $\left.2.659, p=.070, \eta^{2}=.003\right)$. However, lower-income drivers had the lowest expectations of AVs $($ mean $=3.73, \mathrm{SD}=2.01)$ and high-income drivers had the highest expectations of AVs (mean $=$ $4.07, \mathrm{SD}=1.98)$.

\subsection{Driving frequency and expectations of $A V s$}

Participants were divided into two groups according to driving frequency (i.e., sometimes or rarely drive; drive almost every day). There was a significant effect of driving frequency on expectations $\left(\mathrm{F}=10.411, p=.001, \eta^{2}=.006\right)$. Drivers who sometimes or rarely drove had lower expectations of AVs (mean $=3.55, \mathrm{SD}=1.93)$ than people who drove almost every day (mean $=3.97, \mathrm{SD}=$ 2.02).

\subsection{Driving experience and expectations of $A V S$}

Drivers were divided into three groups based on the mean and one standard deviation of their driving experience. The three groups were low (have driven equal to or less than 12 years), medium (have driven more than 12 years and less than 45 years), and high (have driven more than 45 years). Drivers with less than 12 years of driving experience had higher expectations of AVs than the 
other two groups $\left(\mathrm{F}=119.065, p<.001, \eta^{2}=.119\right)$ : low $($ mean $=4.94, \mathrm{SD}=1.68)$ vs. medium $($ mean $=3.89, \mathrm{SD}=2.03 ; p<.001)$; low vs. high $($ mean $=2.79, \mathrm{SD}=1.64 ; p<.001)$. Results are shown in Figure 4.

\subsection{Personality and expectations of $A V S$}

The Big Five personality traits scores were divided into two groups consisting of high or low scores based on their means. Scores above the mean were classified as high and those below the mean were classified as low. ANOVA indicated that there were significant effects of extraversion $(\mathrm{F}=$ $\left.12.328, p<.001, \eta^{2}=.007\right)$, agreeableness $\left(\mathrm{F}=22.256, p<.001, \eta^{2}=.012\right)$, conscientiousness $(\mathrm{F}$ $\left.=44.699, p<0.001, \eta^{2}=.025\right)$, and emotional stability $\left(\mathrm{F}=5.846, p=.016, \eta^{2}=.003\right)$ on expectations. Drivers who were high in each of those personality traits had higher expectations than those who were lower in each personality trait. However, there was no significant difference between people who were high and low in openness to experience $\left(\mathrm{F}=0.250, p=.617, \eta^{2}=.000\right)$.

\subsubsection{Summary of the results}

The findings of this paper can be organized into two overarching results. One, we found significant effects of demographic factors. Results revealed that drivers' expectations of AVs differ greatly by age, gender, ethnicity, education level, marital status, driving frequency, and driving experience. More specifically, drivers who were older, male, non-White non-Hispanics, more highly educated, never married, with a higher drive frequency and with less driving experience were prone to have higher expectations of AVs. Two, this study provides evidence that personality traits do impact AV expectations. In general, drivers who were high in extraversion, agreeableness, conscientiousness, and emotional stability revealed higher expectations of AVs. The next section provides a detailed discussion of the findings and their contributions to the literature.

\section{Discussion}

The goal of this research was to understand whether AV expectations differ by individual differences. Results of this study highlight the significant differences in AV expectations based on age, gender, race and ethnicity, education level, marital status, driving frequency, driving experience, and personality traits. Results of this study can also provide future research with a rich set of factors to explore when predicting AV expectations. 
Our results contribute to the literature in the following ways. First, our findings that AV expectations differ among individuals in a representative sample of U.S. drivers highlight the importance of and extend the literature on individual differences in AV expectations. Prior research discussed individual differences related to public concerns and opinion regarding AVs, and to the best of our knowledge, only one paper discussed expectations related to individual differences; in that paper, men and drivers with higher educational levels had higher expectations of AVs (Schoettle \& Sivak, 2014). Results of our study confirm this assertion that male drivers and those with higher education levels tend to have higher expectations. Further, our study adds to the literature by uncovering other key individual difference factors. More specifically, drivers who were older, female, White non-Hispanic, or rarely involved in driving did not have higher expectations of $\mathrm{AVs}$, whereas drivers who were younger, male, more highly educated, never married, had a higher driving frequency, had less driving experience, and were high in extraversion, agreeableness, emotional stability and conscientiousness were prone to have higher expectations of AVs. In all, the results of this study provide new insights into how individual differences can influence expectations, which act as a predictor of attitudes and behaviors around AVs, including trust, satisfaction, and adoption intention.

Second, the results of this study provide new insights into the relationships between individual differences and AV acceptance. Based on the technology adoption theories (i.e., TAM and UTAUT) and expectation-confirmation theory (ECT), expectations are one determinant of technology adoption (Hossain \& Quaddus, 2012; Kaur \& Rampersad, 2018; Madigan et al., 2017). Expectations that are too low can discourage individuals from ever using a technology, which leads to low technology adoption. However, expectations that are too high can lead to disappointment by setting the bar too high and creating greater discrepancy between expectations and performance, which will also lead to low technology adoption (Lankton et al., 2014; Laumer \& Eckhardt, 2012). In other words, people are less likely to adopt AVs if their expectations are too low but they are also less likely to continue to use them if their expectations are too high and the AV fails to meet those expectations. By understanding which groups are likely to have low or high expectations, we can begin to design interventions to address these issues.

Results of this study found that older drivers tend to have lower expectations compared to younger drivers. This aligned with and helped explain prior literature that suggested that older drivers have negative attitudes toward AV adoption (Hauk et al., 2018; Morris \& Venkatesh, 2000; 
Schoettle \& Sivak, 2014). Because older adults have lower expectations and less interest in driving with AVs, they are less likely to adopt AVs. Similarly, prior literature revealed that male drivers and those who have higher educational levels have a higher acceptance of AVs (Nordhoff et al., 2018; Schoettle \& Sivak, 2014). Our results support and explain this assertion by highlighting the higher expectations that men and people with a higher education level have of AVs. Our results could also explain the relationships between driving experience and AV adoption. Prior research suggested a negative relationship between driving experience and AV adoption (Koul \& Eydgahi, 2018). Our study's findings align with prior research by suggesting that less experienced drivers have higher expectations of AVs.

Our results also found significant effects of extraversion, agreeableness, conscientiousness, and emotional stability on AV expectations. Specifically, our findings suggest that people who are high in these personality traits are prone to have higher expectations of AVs. Previous literature found that drivers with low emotional stability tend to have negative attitudes and lower intentions to use AVs (Kyriakidis et al., 2015; T. Zhang et al., 2020). Our findings aligned with this assertion by suggesting the positive relationship between emotional stability and expectations. To summarize, expectations that are too high or too low can prevent people from having positive attitudes and adopting AVs. Individual differences, including demographics and personality traits, are critical factors to consider because of their impacts on drivers' expectations of AVs. That being said, understanding the effects of individual differences on expectations can help predict and support AV design and adoption.

Finally, this study emphasizes the importance of finding influential factors that can impact AV expectations in terms of expectation calibration. This study provides evidence and examples of how groups with different traits (i.e., individual differences) react differently in terms of their initial expectations, which serves as the baseline for calibration. For example, results showed that men have higher expectations of AVs compared to women. To encourage both men and women to drive with an $\mathrm{AV}$ and decrease disappointment, some effective measures could be done to calibrate their expectations of AVs based on their different initial expectations. Therefore, results of this study demonstrate the need to account for individual differences in AV expectations.

\section{Limitations and Future Research}


Our study has several limitations. First, this study focused on examining drivers' demographics and personality traits. However, individual differences could be found in all psychological characteristics, physical and mental abilities, knowledge, habits, personality, and character traits (Robert, Jr. \& Sykes, 2017; Woodworth \& Marquis, 2014). These include, for instance, cultural differences, religious differences, and motor ability differences. Future studies should examine the relationships between these attributes and expectations of AVs. Second, this study targeted U.S. drivers. Future research could focus on different populations from various countries and investigate whether the results can be replicated. Third, there were large differences in the sample size across groups, which might limit our interpretations of the comparisons across groups. Fourth, this was a cross-sectional observational study, which allowed us to examine whether there were differences but not why there were differences. Future experimental studies could examine causal relationships between individual traits and AV acceptance along with other potential mediation mechanisms. Researchers might also wish to investigate the relationship between AV expectations and AVs' actual adoption, and the relationship between individual differences and different aspects of expectation (i.e., safety and effectiveness expectations). Fifth, individual difference variables were grouped to summarize and test for differences in AV expectations among groups. Despite reaching statistical significance, the effect sizes of education, income, driving frequency, extraversion, emotional stability were quite small. In addition, although detecting subgroups' differences is empirically loosely related to developing a predictive model of AV expectations, it is not clear that the data set we have collected allows us to make strong causal inferences to build valid predictive model. Further research is needed to make causal claims regarding just why these particular demographics and traits were significant. We hope that the results of this paper highlight future directions that others can explore to build valid predictive models. Finally, this study did not consider the participants' previous experiences with AVs when investigating the relationship between individual differences and AV expectations. Although the level 5 fully automated vehicles are not available on the market, participants might have experienced AV-related technology (e.g., AV simulator and virtual AV platforms) before participating in the survey. Individuals who had prior experiences could have different AV expectations from those who had no experience. While we assume all subjects had roughly similar AV experience, future research could investigate whether previous AV-related technology experience influences people's expectations of AVs. In all, more research is needed to investigate AV expectations. 


\section{Conclusion}

This study examined U.S. drivers' expectations of AVs from the perspective of individual differences. Findings in this study emphasize the importance of individual differences, including demographics and personality, on understanding expectations of AVs. More specifically, higher expectations are more often generated by drivers who are younger, male, non-White non-Hispanic, with higher education, never married, with a higher frequency of driving, less driving experience, and who are high in extraversion, agreeableness, emotional stability, and conscientiousness. The results of this study provide a basis for conducting future research related to expectations and AVs. Our results have important implications on the future design of AVs. 


\section{References}

Agarwal, R., \& Prasad, J. (1999). Are individual differences germane to the acceptance of new information technologies? Decision Sciences, 30(2), 361-391.

Allport, G. W., \& Odbert, H. S. (1936). Trait-names: A psycho-lexical study. Psychological Monographs, 47(1), i.

Alshare, K., Grandon, E., \& Miller, D. (2004). Antecedents of computer technology usage: Considerations of the technology acceptance model in the academic environment. Journal of Computing Sciences in Colleges, 19(4), 164-180.

Ayoub, J., Zhou, F., Bao, S., \& Yang, X. J. (2019). From manual driving to automated driving: A review of 10 years of AutoUI. Paper presented at the Proceedings of the 11th International Conference on Automotive User Interfaces and Interactive Vehicular Applications.

Azevedo-Sa, H., Zhao, H., Esterwood, C., Yang, X.J., Tilbury, D.M., Robert, L.P., 2021. How internal and external risks affect the relationships between trust and driver behavior in automated driving systems. Transportation Research Part C: Emerging Technologies 123, 102973. https://doi.org/10.1016/j.trc.2021.102973

Azevedo-Sa, H., Jayaraman, S.K., Yang, X.J., Robert, L.P., Tilbury, D.M., 2020. Context-Adaptive Management of Drivers' Trust in Automated Vehicles. IEEE Robotics and Automation Letters 5, 6908-6915. https://doi.org/10.1109/LRA.2020.3025736

Bansal, P., \& Kockelman, K. M. (2017). Forecasting Americans' long-term adoption of connected and autonomous vehicle technologies. Transportation Research Part A: Policy and Practice, 95, 49-63.

Bhattacherjee, A. (2001). Understanding information systems continuance: An expectation-confirmation model. MIS Quarterly, 25(3), 351-370.

Biondi, F., Alvarez, I., \& Jeong, K.-A. (2019). Human-vehicle cooperation in automated driving: A multidisciplinary review and appraisal. International Journal of Human-Computer Interaction, 35(11), 932-946.

Boas, T. C., Christenson, D. P., \& Glick, D. M. (2018). Recruiting large online samples in the United States and India: Facebook, Mechanical Turk, and Qualtrics. Political Science Research and Methods, 1-19.

Borup, M., Brown, N., Konrad, K., \& Van Lente, H. (2006). The sociology of expectations in science and technology. Technology Analysis \& Strategic Management, 18(3-4), 285-298.

Brown, S. A., Venkatesh, V., \& Goyal, S. (2012). Expectation confirmation in technology use. Information Systems Research, 23(2), 474-487.

Carley, S., Krause, R. M., Lane, B. W., \& Graham, J. D. (2013). Intent to purchase a plug-in electric vehicle: A survey of early impressions in large US cites. Transportation Research Part D: Transport and Environment, 18, 39-45.

Choi, J. K., \& Ji, Y. G. (2015). Investigating the importance of trust on adopting an autonomous vehicle. International Journal of Human-Computer Interaction, 31(10), 692-702.

Clark, B., Parkhurst, G., \& Ricci, M. (2016). Understanding the socioeconomic adoption scenarios for autonomous vehicles: A literature review.

Cooper, C. (2002). Individual differences (Vol. 2). London: Arnold.

Detjen, H., Faltaous, S., Pfleging, B., Geisler, S., \& Schneegass, S. (2021). How to increase automated vehicles' acceptance through in-vehicle interaction design: A review. International Journal of Human-Computer Interaction, 37(4), 308-330.

Digman, J. M. (1997). Higher-order factors of the Big Five. Journal of Personality and Social Psychology, 73(6), 1246.

Du, N., Haspiel, J., Zhang, Q., Tilbury, D., Pradhan, A. K., Yang, X. J., \& Robert Jr., L. P. (2019). Look who’s talking now: Implications of AV's explanations on driver's trust, AV preference, anxiety and mental workload. Transportation Research Part C: Emerging Technologies, 104, 428-442.

Du, N., Zhou, F., Pulver, E.M., Tilbury, D.M., Robert, L.P., Pradhan, A.K., Yang, X.J., 2020. Predicting driver takeover performance in conditionally automated driving. Accident Analysis \& Prevention 148, 105748. https://doi.org/10.1016/j.aap.2020.105748

Esterwood, C., Essenmacher, K., Yang, H., Zeng, F., Robert, L.P., 2021a. A Meta-Analysis of Human Personality and Robot Acceptance in Human-Robot Interaction, in: Proceedings of the $2021 \mathrm{CHI}$ Conference on Human Factors in Computing Systems. Presented at the CHI '21: CHI Conference on Human Factors in Computing Systems, ACM, Yokohama Japan, pp. 1-18. https://doi.org/10.1145/3411764.3445542 
Esterwood, C., Yang, X.J., Robert, L.P., 2021. Barriers to AV Bus Acceptance: A National Survey and Research Agenda. International Journal of Human-Computer Interaction 1-13. https://doi.org/10.1080/10447318.2021.1886485

Favarò, F.M., Eurich, S.O., Rizvi, S.S., 2019. "Human" Problems in Semi-Autonomous Vehicles: Understanding Drivers' Reactions to Off-Nominal Scenarios. International Journal of Human-Computer Interaction 35, 956-971. https://doi.org/10.1080/10447318.2018.1561784

Frison, A.-K., Aigner, L., Wintersberger, P., Riener, A., 2018. Who is Generation A? Investigating the Experience of Automated Driving for Different Age Groups, in: Proceedings of the 10th International Conference on Automotive User Interfaces and Interactive Vehicular Applications, AutomotiveUI '18. Association for Computing Machinery, New York, NY, USA, pp. 94-104. https://doi.org/10.1145/3239060.3239087

Harrison, A. W., \& Rainer, Jr., R. K. (1992). The influence of individual differences on skill in end-user computing. Journal of Management Information Systems, 9(1), 93-111.

Hauk, N., Hüffmeier, J., \& Krumm, S. (2018). Ready to be a silver surfer? A meta-analysis on the relationship between chronological age and technology acceptance. Computers in Human Behavior, 84, 304-319.

Häuslschmid, R., von Buelow, M., Pfleging, B., \& Butz, A. (2017). Supporting trust in autonomous driving. Paper presented at the Proceedings of the 22nd International Conference on Intelligent User Interfaces.

Hossain, M. A., \& Quaddus, M. (2012). Expectation-confirmation theory in information system research: A review and analysis. In Information Systems Theory (pp. 441-469). New York: Springer.

Howard, D., \& Dai, D. (2014). Public perceptions of self-driving cars: The case of Berkeley, California. Paper presented at the Transportation Research Board 93rd Annual Meeting.

Im, S., Bayus, B. L., \& Mason, C. H. (2003). An empirical study of innate consumer innovativeness, personal characteristics, and new-product adoption behavior. Journal of the Academy of Marketing Science, 31(1), 61-73.

Jayaraman, S., Creech, C., Dawn, T., Yang, X. J., Pradhan, A., Tsui, K., \& Robert, L. (2019, November 28). Pedestrian trust in automated vehicles: Role of traffic signal and AV driving behavior. Frontiers in Robotics and AI. Retrieved from https://www.frontiersin.org/articles/10.3389/frobt.2019.00117/full

Kaur, K., \& Rampersad, G. (2018). Trust in driverless cars: Investigating key factors influencing the adoption of driverless cars. Journal of Engineering and Technology Management, 48, 87-96.

Körber, M., Baseler, E., \& Bengler, K. (2018). Introduction matters: Manipulating trust in automation and reliance in automated driving. Applied Ergonomics, 66, 18-31.

Koul, S., \& Eydgahi, A. (2018). Utilizing technology acceptance model (TAM) for driverless car technology adoption. Journal of Technology Management \& Innovation, 13(4), 37-46.

Kyriakidis, M., Happee, R., \& de Winter, J. C. (2015). Public opinion on automated driving: Results of an international questionnaire among 5,000 respondents. Transportation Research Part F: Traffic Psychology and Behaviour, 32, 127-140.

Lankton, N., McKnight, D. H., \& Thatcher, J. B. (2014). Incorporating trust-in-technology into Expectation Disconfirmation Theory. The Journal of Strategic Information Systems, 23(2), 128-145.

Laumer, S., \& Eckhardt, A. (2012). Why do people reject technologies: A review of user resistance theories. In: Y. Dwivedi, M. Wade, S. Schneberger (Eds.), Information Systems Theory (Vol. 28). New York: Springer.

Lee, C., \& Coughlin, J. F. (2015). PERSPECTIVE: Older adults' adoption of technology: An integrated approach to identifying determinants and barriers. Journal of Product Innovation Management, 32(5), 747-759.

Lee, H.-J., Jeong Cho, H., Xu, W., \& Fairhurst, A. (2010). The influence of consumer traits and demographics on intention to use retail self-service checkouts. Marketing Intelligence \& Planning, 28(1), 46-58.

Ma, Q., Chan, A. H., \& Chen, K. (2016). Personal and other factors affecting acceptance of smartphone technology by older Chinese adults. Applied Ergonomics, 54, 62-71.

Ma, Q., Chan, A.H.S., Teh, P.-L., 2021. Insights into Older Adults' Technology Acceptance through Meta-Analysis. International Journal of Human-Computer Interaction 37, 1049-1062. https://doi.org/10.1080/10447318.2020.1865005

Madigan, R., Louw, T., Wilbrink, M., Schieben, A., \& Merat, N. (2017). What influences the decision to use automated public transport? Using UTAUT to understand public acceptance of automated road transport systems. Transportation Research Part F: Traffic Psychology and Behaviour, 50, 55-64.

Mamun, M. R. A., Senn, W. D., Peak, D. A., Prybutok, V. R., \& Torres, R. A. (2020). Emotional satisfaction and IS continuance behavior: Reshaping the expectation-confirmation model. International Journal of HumanComputer Interaction, 36(15), 1437-1446. 
Maruping, L. M., Bala, H., Venkatesh, V., \& Brown, S. A. (2017). Going beyond intention: Integrating behavioral expectation into the unified theory of acceptance and use of technology. Journal of the Association for Information Science and Technology, 68(3), 623-637.

McCrae, R. R., \& Costa, Jr., P. T. (1997). Conceptions and correlates of openness to experience. In R. Hogan, J. Johnson, \& S. Briggs (Eds.), Handbook of Personality Psychology (pp. 825-847): New York: Elsevier.

Merat, N., \& Jamson, A. H. (2009). How do drivers behave in a highly automated car? Retrieved from https://www.researchgate.net/publication/237622858_How_do_drivers_behave_in_a_highly_automated_ca $r$

Meuter, M. L., Bitner, M. J., Ostrom, A. L., \& Brown, S. W. (2005). Choosing among alternative service delivery modes: An investigation of customer trial of self-service technologies. Journal of Marketing, 69(2), 61-83.

Meyer, J., Becker, H., Bösch, P. M., \& Axhausen, K. W. (2017). Autonomous vehicles: The next jump in accessibilities? Research in Transportation Economics, 62, 80-91.

Michalco, J., Simonsen, J. G., \& Hornbæk, K. (2015). An exploration of the relation between expectations and user experience. International Journal of Human-Computer Interaction, 31(9), 603-617.

Mitchell, U. A., Chebli, P. G., Ruggiero, L., \& Muramatsu, N. (2019). The digital divide in health-related technology use: The significance of race/ethnicity. The Gerontologist, 59(1), 6-14.

Morris, M. G., \& Venkatesh, V. (2000). Age differences in technology adoption decisions: Implications for a changing work force. Personnel Psychology, 53(2), 375-403.

Nordhoff, S., De Winter, J., Kyriakidis, M., Van Arem, B., \& Happee, R. (2018). Acceptance of driverless vehicles: Results from a large cross-national questionnaire study. Journal of Advanced Transportation. Retrieved from

https://www.researchgate.net/publication/323907099_Acceptance_of_Driverless_Vehicles_Results_from_a Large_Cross-National_Questionnaire_Study

Nordhoff, S., van Arem, B., \& Happee, R. (2016). Conceptual model to explain, predict, and improve user acceptance of driverless podlike vehicles. Transportation Research Record, 2602(1), 60-67.

Oliver, R. L. (1980). A cognitive model of the antecedents and consequences of satisfaction decisions. Journal of Marketing Research, 17(4), 460-469.

O'Neill, T. A., \& Allen, N. J. (2011). Personality and the prediction of team performance. European Journal of Personality, 25(1), 31-42.

Peeters, M. A., Van Tuijl, H. F., Rutte, C. G., \& Reymen, I. M. (2006). Personality and team performance: A metaanalysis. European Journal of Personality, 20(5), 377-396.

Petersen, L., Robert, L., Yang, X.J., Tilbury, D., 2019. Situational Awareness, Driver's Trust in Automated Driving Systems and Secondary Task Performance. SAE Intl. J CAV 2, 12-02-02-0009. https://doi.org/10.4271/1202-02-0009

Pettifor, H., Wilson, C., Axsen, J., Abrahamse, W., \& Anable, J. (2017). Social influence in the global diffusion of alternative fuel vehicles-A meta-analysis. Journal of Transport Geography, 62, 247-261.

Ricci, M., n.d. Understanding the socioeconomic adoption scenarios for autonomous vehicles: A literature review.

Ro, Y., \& Ha, Y. (2019). A factor analysis of consumer expectations for autonomous cars. Journal of Computer Information Systems, 59(1), 52-60.

Robert, L. P. (2018). Personality in the Human Robot Interaction Literature: A Review and Brief Critique, Proceedings of the 24th Americas Conference on Information Systems, Aug 16-18, New Orleans, LA

Robert, L., Alahmad, R., Esterwood, C., Kim, S., You, S., \& Zhang, Q. (2020). A review of personality in humanrobot interactions. Foundations and Trends in Information Systems, 4(2), 107-112.

Robert, L. P. (2019). Are automated vehicles safer than manually driven cars? AI \& SOCIETY, 34(3), 687-688.

Robert, Jr., L. P., \& Sykes, T. A. (2017). Extending the concept of control beliefs: Integrating the role of advice networks. Information Systems Research, 28(1), 84-96.

Rödel, C., Stadler, S., Meschtscherjakov, A., \& Tscheligi, M. (2014). Towards autonomous cars: The effect of autonomy levels on acceptance and user experience. Paper presented at the Proceedings of the 6th International Conference on Automotive User Interfaces and Interactive Vehicular Applications.

Sarkar, S., \& Khare, A. (2019). Influence of expectation confirmation, network externalities, and flow on use of mobile shopping apps. International Journal of Human-Computer Interaction, 35(16), 1449-1460.

Schoettle, B., \& Sivak, M. (2014). A survey of public opinion about autonomous and self-driving vehicles in the U.S., the U.K., and Australia. Paper presented at the 2014 International Conference on Connected Vehicles and Expo (ICCVE). 
Sovacool, B. K., Kester, J., Noel, L., \& de Rubens, G. Z. (2019). Income, political affiliation, urbanism and geography in stated preferences for electric vehicles (EVs) and vehicle-to-grid (V2G) technologies in Northern Europe. Journal of Transport Geography, 78, 214-229.

Tan, H., Chen, C., Hao, Y., 2021. How People Perceive and Expect Safety in Autonomous Vehicles: An Empirical Study for Risk Sensitivity and Risk-related Feelings. International Journal of Human-Computer Interaction 37, 340-351. https://doi.org/10.1080/10447318.2020.1860515

Thong, J. Y., Hong, S.-J., \& Tam, K. Y. (2006). The effects of post-adoption beliefs on the expectation-confirmation model for information technology continuance. International Journal of Human-Computer Studies, 64(9), 799-810.

Triplett, T., Santos, R., Rosenbloom, S., \& Tefft, B. (2016). American driving survey: 2014-2015. Washington, DC: AAA Foundation.

Tussyadiah, I. P., Zach, F. J., \& Wang, J. (2017). Attitudes toward autonomous on demand mobility system: The case of self-driving taxi. In Information and Communication Technologies in Tourism 2017 (pp. 755-766). New York: Springer.

Van Ryzin, G. G. (2004). Expectations, performance, and citizen satisfaction with urban services. Journal of Policy Analysis and Management, 23(3), 433-448.

Venkatesh, V., Sykes, T. A., \& Venkatraman, S. (2014). Understanding e-government portal use in rural India: role of demographic and personality characteristics. Information Systems Journal, 24(3), 249-269.

Waller, P. F. (1991). The older driver. Human Factors, 33(5), 499-505.

Woodworth, R. S., \& Marquis, D. G. (2014). Psychology (psychology revivals): A study of mental life. London: Psychology Press.

Yoon, Y. J., Park, D. E., \& Park, S. E. (2016). Comparison of interaction behavior analysis between for [sic] elderly drivers and young drivers in terms of driving experience. In Proceedings of HCI Korea (pp. 259-265).

Zhang, Q., Yang, X.J., Robert, L.P., 2021. Drivers' Age and Automated Vehicle Explanations. Sustainability 13, 1948. https://doi.org/10.3390/su13041948

Zhang, T., Tao, D., Qu, X., Zhang, X., Zeng, J., Zhu, H., \& Zhu, H. (2020). Automated vehicle acceptance in China: Social influence and initial trust are key determinants. Transportation Research Part C: Emerging Technologies, 112, 220 


\section{About the Authors:}

\section{Qiaoning Zhang}

Qiaoning Zhang is a Ph.D student at the University of Michigan's School of Information and is a member of the Michigan Autonomous Vehicle Research Intergroup Collaboration (MAVRIC). She obtained her Master degree in Industrial and Operations Engineering from University of Michigan.

\section{Jessie Yang}

X. Jessie Yang is an Assistant Professor at the Department of Industrial and Operations Engineering, University of Michigan Ann Arbor. She obtained her $\mathrm{PhD}$ in Mechanical and Aerospace Engineering (Human Factors) from Nanyang Technological University, Singapore in 2014.

\section{Lionel P. Robert Jr.}

Lionel P. Robert Jr. an Associate Professor in the School of Information and core faculty member of the Robotics Institute both at the University of Michigan. He is the director of the Michigan Autonomous Vehicle Research Intergroup Collaboration (MAVRIC) and an Association for Information Systems Distinguished Member. 
Table 1

Demographics

\begin{tabular}{|c|c|c|c|c|}
\hline $\begin{array}{l}\text { Demographic } \\
\text { Characteristics }\end{array}$ & & $\begin{array}{l}\text { Population } \\
\text { Percentage }\end{array}$ & $\begin{array}{c}\text { Sample } \\
\text { Percentage }\end{array}$ & Number \\
\hline \multirow{2}{*}{ Age } & Younger Driver (18-54) & $63.8 \%$ & $63.7 \%$ & 282 \\
\hline & Older Driver $(55+)$ & $36.2 \%$ & $36.3 \%$ & 161 \\
\hline \multirow{2}{*}{ Gender } & Male & $49.0 \%$ & $49.0 \%$ & 217 \\
\hline & Female & $51.0 \%$ & $51.0 \%$ & 226 \\
\hline \multirow{4}{*}{ Region } & Northeast & $17.0 \%$ & $16.5 \%$ & 73 \\
\hline & West & $24.0 \%$ & $23.5 \%$ & 104 \\
\hline & South & $38.0 \%$ & $40.1 \%$ & 178 \\
\hline & Midwest & $21.0 \%$ & $19.6 \%$ & 87 \\
\hline \multirow{4}{*}{ Ethnicity } & White non-Hispanic & $65.3 \%$ & $65.2 \%$ & 289 \\
\hline & $\begin{array}{l}\text { Black/African American non- } \\
\text { Hispanic }\end{array}$ & $14.1 \%$ & $14.0 \%$ & 62 \\
\hline & Hispanic & $14.8 \%$ & $14.9 \%$ & 66 \\
\hline & Other & $5.8 \%$ & $5.8 \%$ & 26 \\
\hline \multirow{3}{*}{ Education $^{\mathrm{a}}$} & $\begin{array}{c}\text { Less than high school or high } \\
\text { school/GED }\end{array}$ & $39.0 \%$ & $23.6 \%$ & 97 \\
\hline & Some college & $21.0 \%$ & $24.3 \%$ & 100 \\
\hline & College graduate & $40.0 \%$ & $52.1 \%$ & 214 \\
\hline \multirow{5}{*}{ Marital Status ${ }^{\mathrm{b}}$} & Never married & $23.5 \%$ & $32.3 \%$ & 143 \\
\hline & Married & $51.7 \%$ & $42.0 \%$ & 186 \\
\hline & Living with partner & $6.5 \%$ & $5.4 \%$ & 24 \\
\hline & Widowed & $7.5 \%$ & $4.5 \%$ & 20 \\
\hline & Divorced/separated & $10.8 \%$ & $15.8 \%$ & 70 \\
\hline \multirow{3}{*}{ Income } & Low Income $(<\$ 35 \mathrm{k} / \mathrm{yr})$ & $\mathrm{N} / \mathrm{A}$ & $25.1 \%$ & 111 \\
\hline & Medium Income (\$35k-\$100k/yr) & $\mathrm{N} / \mathrm{A}$ & $62.8 \%$ & 278 \\
\hline & High Income $(>\$ 100 \mathrm{k} / \mathrm{yr})$ & $\mathrm{N} / \mathrm{A}$ & $12.2 \%$ & 54 \\
\hline \multirow{2}{*}{ Driving Frequency } & Drives almost every day & $68.5 \%$ & $84.0 \%$ & 372 \\
\hline & Drives sometimes or rarely & $18.1 \%$ & $16.0 \%$ & 71 \\
\hline \multirow{3}{*}{ Driving Experience ${ }^{\mathfrak{c}}$} & Low $(<=12$ yrs $)$ & $\mathrm{N} / \mathrm{A}$ & $21.0 \%$ & 93 \\
\hline & Medium (13-45 yrs) & $\mathrm{N} / \mathrm{A}$ & $58.0 \%$ & 257 \\
\hline & $\operatorname{High}(=>46$ yrs $)$ & $\mathrm{N} / \mathrm{A}$ & $20.3 \%$ & 90 \\
\hline \multirow{2}{*}{ Extraversion } & Solitary/reserved & $\mathrm{N} / \mathrm{A}$ & $57.8 \%$ & 256 \\
\hline & Outgoing/energetic & $\mathrm{N} / \mathrm{A}$ & $42.2 \%$ & 187 \\
\hline \multirow{2}{*}{ Agreeableness } & Challenging/detached & $\mathrm{N} / \mathrm{A}$ & $43.6 \%$ & 193 \\
\hline & Friendly/compassionate & N/A & $56.4 \%$ & 250 \\
\hline \multirow{2}{*}{ Conscientiousness } & Efficient/organized & N/A & $40.6 \%$ & 180 \\
\hline & Easy-going/careless & $\mathrm{N} / \mathrm{A}$ & $59.4 \%$ & 263 \\
\hline \multirow{2}{*}{ Emotional Stability } & Sensitive/nervous & N/A & $48.3 \%$ & 214 \\
\hline & Secure/confident & N/A & $51.7 \%$ & 229 \\
\hline \multirow{2}{*}{$\begin{array}{l}\text { Openness to } \\
\text { Experience }\end{array}$} & Consistent/cautious & N/A & $53.7 \%$ & 238 \\
\hline & Inventive/curious & $\mathrm{N} / \mathrm{A}$ & $46.3 \%$ & 205 \\
\hline
\end{tabular}


Table 2

Analysis Results

\begin{tabular}{|c|c|c|c|c|c|c|}
\hline \multirow{2}{*}{$\begin{array}{c}\text { Demographic } \\
\text { Characteristics } \\
\end{array}$} & & Number & \multicolumn{4}{|c|}{ Expectations } \\
\hline & & & Mean & St. dev. & $\mathbf{F}$ & $p$ value \\
\hline \multirow{2}{*}{ Age* } & Younger Driver (18-54) & 282 & 4.40 & 1.93 & \multirow{2}{*}{208.856} & \multirow{2}{*}{$<.001$} \\
\hline & Older Driver $(55+)$ & 161 & 3.04 & 1.86 & & \\
\hline \multirow{2}{*}{ Gender* } & Male & 217 & 4.31 & 1.98 & \multirow{2}{*}{69.818} & \multirow{2}{*}{$<.001$} \\
\hline & Female & 226 & 3.52 & 1.96 & & \\
\hline \multirow{4}{*}{ Region } & Northeast & 73 & 3.93 & 2.16 & \multirow{4}{*}{0.657} & \multirow{4}{*}{.578} \\
\hline & West & 104 & 3.79 & 2.03 & & \\
\hline & South & 178 & 3.96 & 1.94 & & \\
\hline & Midwest & 87 & 3.91 & 2.02 & & \\
\hline \multirow{4}{*}{ Ethnicity* } & White non-Hispanic & 289 & 3.68 & 1.99 & \multirow{4}{*}{14.944} & \multirow{4}{*}{$<.001$} \\
\hline & $\begin{array}{c}\text { Black/African American } \\
\text { non-Hispanic }\end{array}$ & 62 & 4.40 & 1.94 & & \\
\hline & Hispanic & 66 & 4.32 & 2.07 & & \\
\hline & Other & 26 & 4.21 & 1.83 & & \\
\hline \multirow{3}{*}{ Education* } & High school or GED & 97 & 3.66 & 2.00 & \multirow{3}{*}{5.192} & \multirow{3}{*}{.001} \\
\hline & Some college & 100 & 3.58 & 2.09 & & \\
\hline & College graduate & 214 & 3.98 & 1.95 & & \\
\hline \multirow{5}{*}{ Marital Status* } & Never married & 143 & 4.47 & 1.84 & \multirow{5}{*}{20.319} & \multirow{5}{*}{$<.001$} \\
\hline & Married & 186 & 3.74 & 2.06 & & \\
\hline & Living with partner & 24 & 3.92 & 2.21 & & \\
\hline & Widowed & 20 & 3.25 & 1.80 & & \\
\hline & Divorced/separated & 70 & 3.37 & 1.94 & & \\
\hline \multirow{3}{*}{ Income } & Low Income $(<\$ 35 \mathrm{k} / \mathrm{yr})$ & 111 & 3.73 & 2.01 & \multirow{3}{*}{2.659} & \multirow{3}{*}{.070} \\
\hline & $\begin{array}{l}\text { Medium Income }(\$ 35 \mathrm{k}- \\
\$ 100 \mathrm{k} / \mathrm{yr})\end{array}$ & 278 & 3.94 & 2.01 & & \\
\hline & $\begin{array}{l}\text { High Income } \\
(>\$ 100 \mathrm{k} / \mathrm{yr})\end{array}$ & 54 & 4.07 & 1.98 & & \\
\hline \multirow{2}{*}{$\begin{array}{l}\text { Driving } \\
\text { Frequency* }\end{array}$} & Drives almost every day & 372 & 3.97 & 2.02 & \multirow[b]{2}{*}{10.411} & \multirow[b]{2}{*}{.001} \\
\hline & $\begin{array}{l}\text { Drives sometimes or } \\
\text { rarely }\end{array}$ & 71 & 3.55 & 1.93 & & \\
\hline & Low ( $\leq 12$ yrs $)$ & 93 & 4.94 & 1.68 & & \\
\hline Exnerience* & Medium (13-45 yrs) & 257 & 3.89 & 2.03 & 119.065 & $<.001$ \\
\hline & $\operatorname{High}(\geq 46$ yrs $)$ & 90 & 2.79 & 1.64 & & \\
\hline Fytrayercion* & Solitary/reserved & 256 & 3.76 & 1.96 & 12328 & $<001$ \\
\hline Exuraversion & Outgoing/energetic & 187 & 4.10 & 2.06 & 12.520 & -.001 \\
\hline A oreeableness* & Challenging/detached & 193 & 3.64 & 2.13 & 22.256 & $<001$ \\
\hline & Friendly/compassionate & 250 & 4.10 & 1.89 & & \\
\hline Concriantiousnecs* & Efficient/organized & 180 & 3.59 & 2.16 & 44699 & $<001$ \\
\hline Conscientiousness" & Easy-going/careless & 263 & 4.12 & 1.88 & 44.099 & $<.001$ \\
\hline Emotional & Sensitive/nervous & 214 & 3.79 & 2.09 & 5.846 & 016 \\
\hline Stability* & Secure/confident & 229 & 4.02 & 1.93 & 0.040 & .010 \\
\hline Openness to & Consistent/cautious & 238 & 3.88 & 1.90 & 0250 & 617 \\
\hline Experience & Inventive/curious & 205 & 3.93 & 2.13 & 0.250 & .011 \\
\hline
\end{tabular}

Note. Asterisks mark the demographic variables that have significant effects on expectations of AVs. 


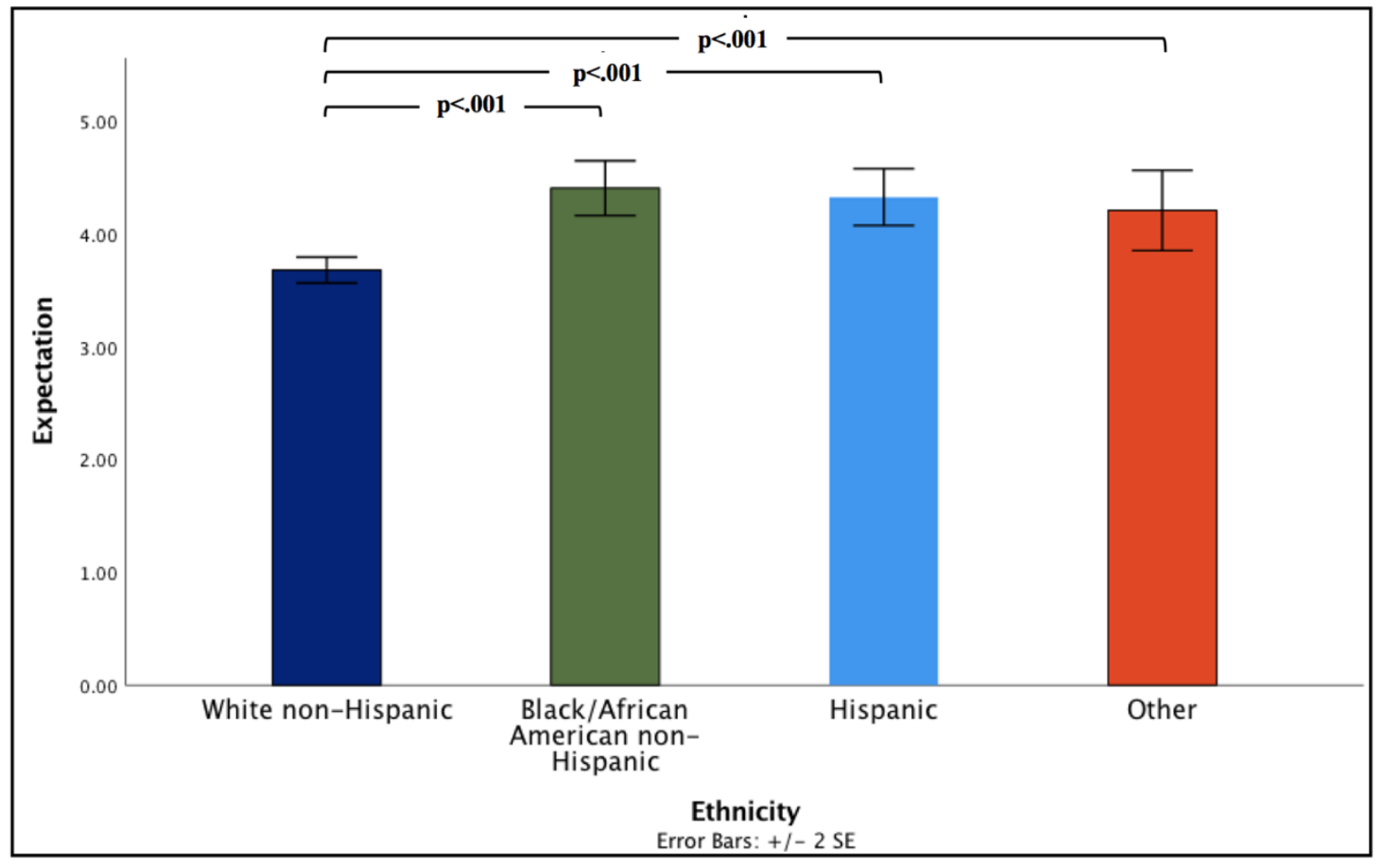

Figure 1. Summary of the responses, by ethnicity, to people's expectations of AVs.

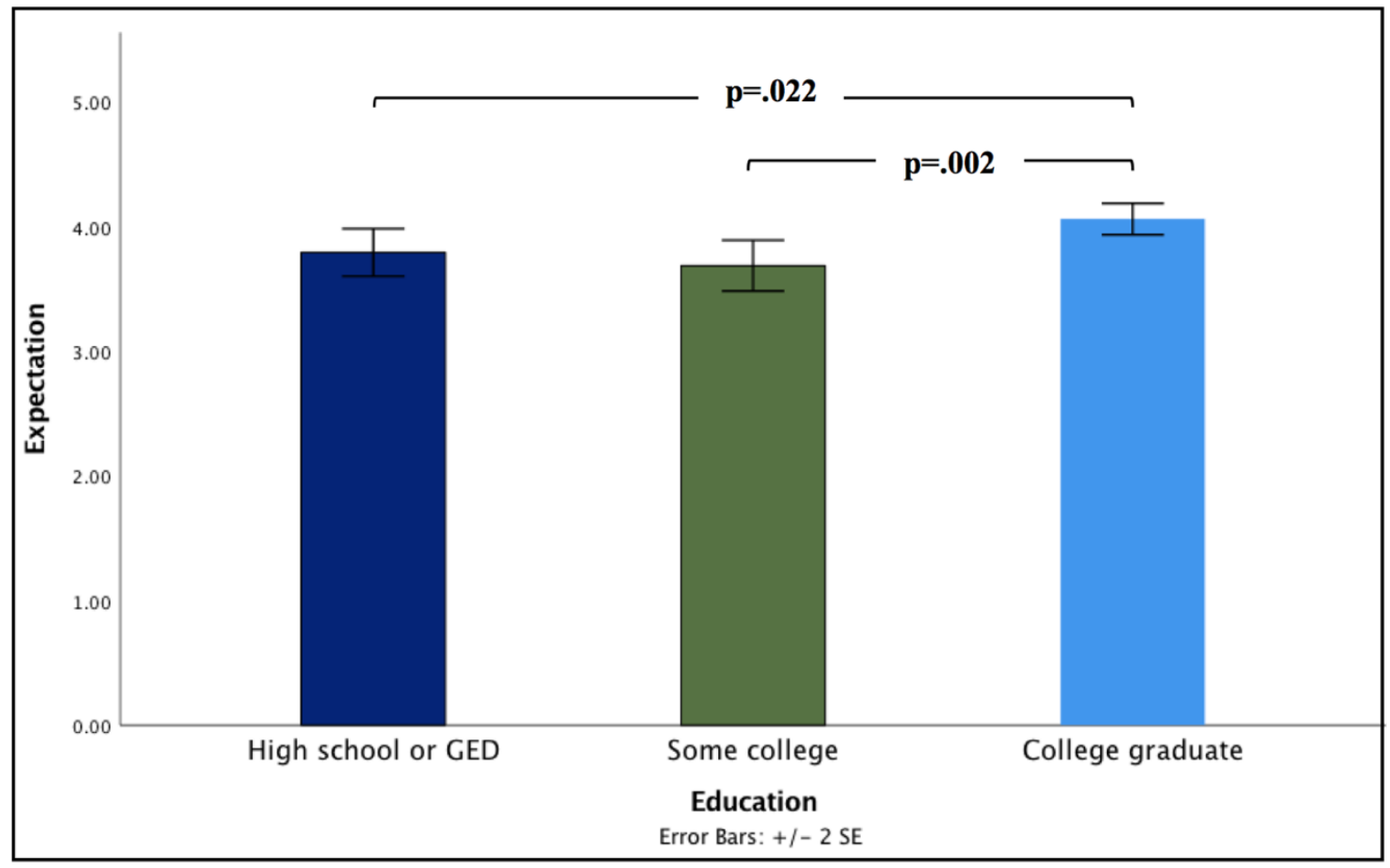

Figure 2. Summary of the responses, by education, to people's expectations of AVs. 


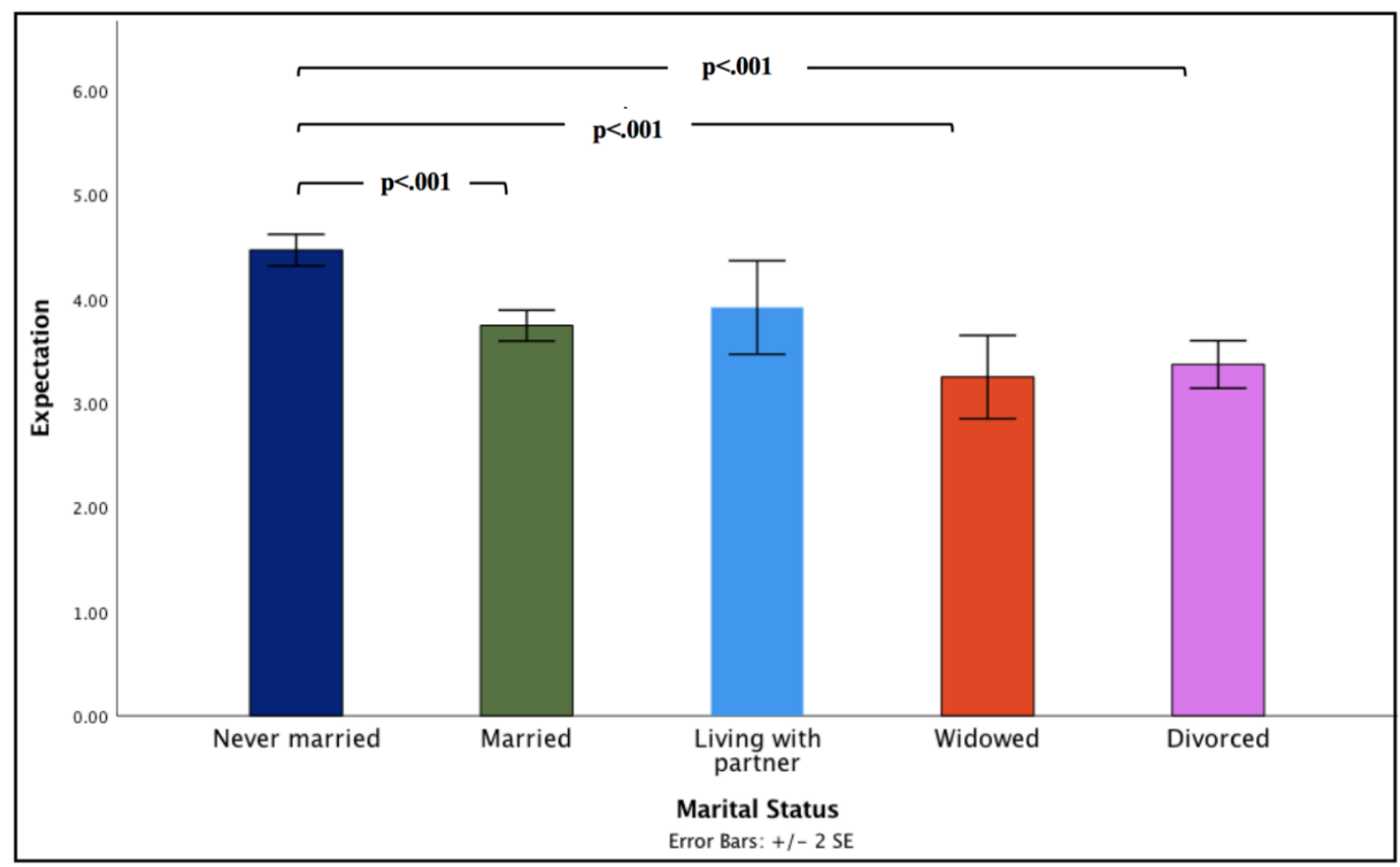

Figure 3. Summary of the responses, by marital status, to people's expectations of AVs.

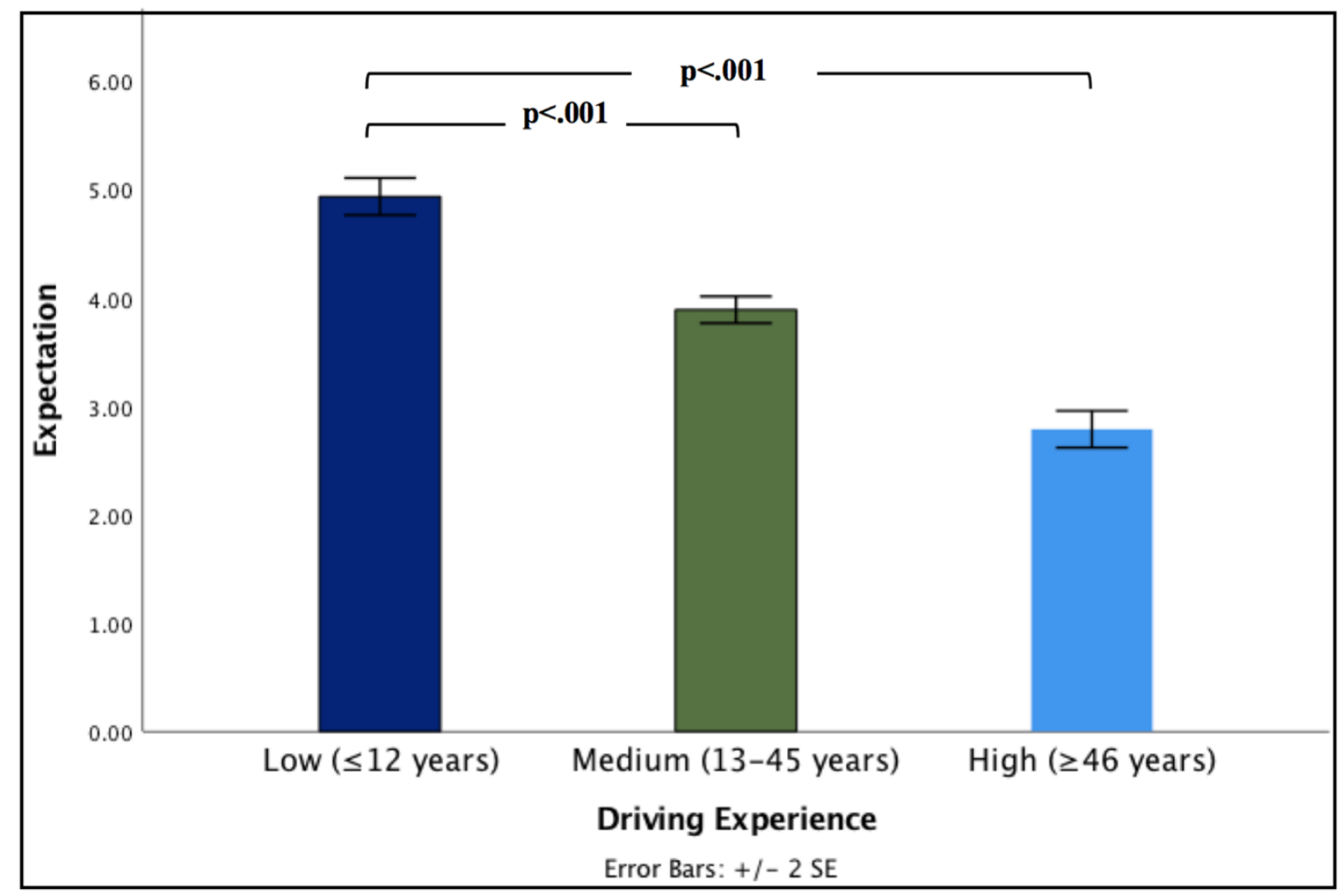

Figure 4. Summary of the responses, by driving experience, to people's expectations of AVs. 